\title{
Tamanho de amostra para avaliar a densidade populacional de percevejos em lavouras de soja
}

\author{
Sample size to evaluate the population density of bugs in soybean field
}

\author{
Claudia Carolina Cabral Antúnez ${ }^{\mathrm{I}}$ Lindolfo $_{\text {Storck }}{ }^{\mathrm{I}^{*}, \mathrm{III}}$ Jerson Vanderlei Carús Guedes ${ }^{\mathrm{II}}$ \\ Alberto Cargnelutti Filho'II Jimmy Walter Rasche Alvarez ${ }^{I}$
}

\section{RESUMO}

\begin{abstract}
O objetivo deste trabalho foi estimar o tamanho de amostra para medir a densidade populacional de espécies de percevejos, variando métodos de coleta, altitude e cultivares de soja. Foram utilizadas 100 lavouras de soja, distribuídas em nove municipios da região central do Rio Grande do Sul, em três safras agrícolas (2010/2011, 2011/2012, 2012/2013). Em cada lavoura, foram demarcados 30 pontos distantes em 20 metros entre si. Em cada ponto, foram coletados percevejos (adultos + ninfas) das espécies Dichelops sp., Piezodorus guildinii e Euchistus heros, por meio dos métodos pano-de-batida largo e rede entomológica, totalizando 6.000 coletas. Para cada lavoura, método de coleta e espéciede percevejo, foram estimadas a média da densidade populacional e o tamanho de amostra por meio de reamostragem. $O$ tamanho de amostra (número de pontos) para determinar a densidade populacional de percevejos varia com o método de coleta e a densidade populacional. Usando o pano-de-batida largo para a coleta de percevejos em soja, para um erro de estimação (amplitude do intervalo de credibilidade) igual a dois percevejos e densidade populacional na classe de 1,5 a 2,0 percevejos $m^{-2}$ o tamanho de amostra é 13, 77 e 15, respectivamente, para as espécies Dichelops sp., P. guildinii e E. heros.
\end{abstract}

Palavras-chave: Glycine max, método de coleta, pragas de soja, amostragem, reamostragem, dimensionamento amostral.

\section{ABSTRACT}

The objective was to estimate the sample size to evaluate the population density of bugs species, varying collection methods, altitude and soybean cultivars. There were used 100 soybean fields, distributed in nine districts of the central region of Rio Grande do Sul on three growing seasons (2010/2011, 2011/2012, 2012/2013). In each field, 30 equidistant points were marked at 20 meters. Bugs (adults and nymphs) of Dichelops sp., Piezodorus guildinii and Euchistus heros species were collected at each point using beat cloth and sweep net, totaling 6,000 collections. For each field, collection method and bug speciesit was estimated average population density, and sample size by resampling. The sample size (number of points) to estimate the population density of bugs vary with the species, altitude, soybean cultivar and the collection method. Using the beat cloth method to collect bugs in soybean, for an estimate error equal to two bugs and population density at class of 1.5 to 2.0 bugs $\mathrm{m}^{-2}$, the number of sampling points are 13, 77 and 15, respectively, for Dichelops sp., P. guildinii and $\boldsymbol{E}$. heros.

Key words: Glycine max, collection method, soybean pests, sampling, resampling, sample dimension.

\section{INTRODUÇÃO}

A cultura da soja [Glycine $\max$ (L.) Merr.] é de grande importância econômica para o Brasil. Nos últimos anos, houve aumento no interesse dos agricultores pela cultura, devido ao aumento do preço do grão no mercado mundial. Esse fato fez com que a área cultivada no Brasil chegasse a 31,5 milhões de ha em 2014/2015 (CONAB, 2015). A produtividade de grãos de soja está relacionada com a ocorrência de insetos-pragas, que causam perdas e aumentam os custos de produção. Os percevejos, alimentando-se dos grãos, reduzem o peso e a qualidade destes (CORRÊA-FERREIRA, 2005). Assim, é importante monitorar as lavouras quanto

\footnotetext{
'Facultad de Ciencias Agrarias (FCA), Universidad Nacional de Asunción (UNA), San Lorenzo, Paraguay.

IIUniversidade Federal de Santa Maria (UFSM), Av. Roraima, 1000, Camobi, 97105-900, Santa Maria, RS, Brasil. E-mail: lindolfostorck@gmail.com. *Autor para correspondência.

IIIUniversidade Tecnológica Federal do Paraná (UTFPR), Pato Branco, PR, Brasil. 
à densidade populacional desses insetos durante o estágio de desenvolvimento da cultura.

Os programas de manejo integrado de pragas se baseiam em métodos de coleta (CULLEN et al., 2000; FARIAS et al., 2001; GUEDES et al., 2006; STÜRMER et al., 2012). Para implementar um manejo integrado de pragas racional, é necessário conhecer o método de coleta e o tamanho de amostra, que permitam estimar a densidade populacional da praga, com erro de estimação conhecido. Métodos de coleta por pano-de-batida, pano vertical, rede entomológica e contagem absoluta podem ser usados para a estimativa da densidade populacional de pragas e inimigos naturais de soja. O pano-de-batida largo é um dos métodos de coleta mais recomendado (CORRÊA-FERREIRA, 2012; STÜRMER et al., 2012). Por outro lado, a rede entomológica é o método mais usado para coleta de insetos quando há presença de maior número de ordens ou de insetos com maior agilidade (LARA et al., 2008).

$\mathrm{Na}$ cultura de soja, há indicações de tamanho de amostra igual a oito pontos amostrais (GALLO et al., 2002) e dez pontos (CORRÊAFERREIRA, 2012). Entretanto, foi constatado (STÜRMER et al., 2014a) que são necessários 36 pontos amostrais, para um erro de estimação (semiamplitude do intervalo de confiança; $1-\alpha=0,95$ ) igual a $30 \%$ da média estimada, para determinar a densidade populacional de percevejos pelo método de coleta pano-de-batida vertical, numa lavoura de 6,1ha, nos estágios fenológicos finais da soja (R6, R7.1, R7.3 e R8.2).

Existe a necessidade de informações que possibilitem recomendações de métodos de coleta e respectivo tamanho de amostra para as principais pragas da cultura de soja, em condições ambientais variadas. $\mathrm{O}$ objetivo deste trabalho foi determinar o tamanho de amostra para estimar a densidade populacional de espécies de percevejos, variando métodos de coleta, altitude e cultivar de soja.

\section{MATERIAL E MÉTODOS}

O estudo foi realizado em 100 lavouras comerciais de soja, mantidas com manejo controle mínimo de pragas, variando as cultivares e os estágios de desenvolvimento. Os municípios do estado do Rio Grande do Sul onde as lavouras se localizaram foram: São Sepé, Formigueiro, Restinga Seca, Itaara, Júlio de Castilhos, São Pedro do Sul, Dilermando de Aguiar, Santa Maria e São Gabriel. Nesses locais, nas safras de 2010/11, 2011/12 e 2012/13, foram avaliadas 41, 30 e 29 lavouras, respectivamente.
Em cada lavoura, foram demarcados 30 pontos, equidistantes em 20 metros, sendo 10 pontos em cada três fileiras de soja. Em cada um dos 30 pontos, foram realizadas as coletas dos insetos presentes, empregando dois métodos de coleta: panode-batida largo e rede entomológica, uma em cada lado (esquerdo e direito) dos pontos.

O pano-de-batida largo é um pano branco de $1,0 \mathrm{~m}$ de comprimento por 1,0m de largura, com as bordas inseridas em cabo de madeira (STÜRMER et al., 2012). Esse pano é colocado desde a base de uma fileira de soja até a outra fileira e as plantas de um lado são batidas vigorosamente sobre o pano, de modo a que os insetospragas presentes fiquem depositados no pano. Nesse caso, a área amostrada em cada ponto é igual a $0,5 \mathrm{~m}^{2}$. A rede entomológica é constituída de um cabo de madeira de $1,0 \mathrm{~m}$, com aro de $0,35 \mathrm{~cm}$ de diâmetro. As coletas dos insetos com a rede entomológicasão realizadas por meio de cinco passadas da rede sobre as plantas de duas fileiras e um metro linear, seguindo a linha imaginária de um "oito aberto" (PANIZZI \& CORRÊA-FERREIRA, 1978), resultando numa área de $1,0 \mathrm{~m}^{2}$.

O número de percevejos (adultos e ninfas) das espécies Dichelops sp., Piezodorus guildinii (Westwood) e Euchistus heros (F.), foi anotado em planilha eletrônica e ajustado para $1,0 \mathrm{~m}^{2}$, com a respectiva identificação da lavoura, ponto amostral e método de coleta.

Para cada uma das 100 lavouras, para cada método de coleta e para cada espécie, foi estimada a média e determinado o tamanho de amostra. $\mathrm{O}$ tamanho de amostra foi obtido pelo método de reamostragem (EFRON, 1979; FERREIRA, 2005; STORCK et al., 2012). Esse método é adequado, tendo em vista que os valores do número de insetos por ponto (densidade populacional), em geral, não seguem distribuição de probabilidade conhecida ou não é a mesma para os diferentes ambientes, método de coleta e espécies (BARBOSA, 2001). Para isso, usando os valores do número de percevejos, de cada espécie, obtidos nos 30 pontos da lavoura, foram geradas 2.000 reamostras $(\mathrm{J})$, com reposição, e calculada a média de cada reamostra. Estas 2.000 estimativas de médias foram ordenadas para a identificação do quantil 0,025 como limite inferior (LI) e do quantil 0,975 como limite superior (LS). Os valores de LI e LS correspondem à estimativa do intervalo de confiança bootstrap percentílico (IC), com taxa de erro igual a 0,05 . O mesmo procedimento, 2.000 reamostras com reposição, foi repetido para os tamanhos de amostra igual a $\mathrm{k}(\mathrm{k}=3,4, \ldots, 200) \mathrm{em}$ cada lavoura. Para cada valor de k, as 2.000 médias, obtidas com as 2.000 reamostras de tamanho $\mathrm{k}$, foram ordenadas para a identificação dos LI(k) e LS(k). Os 
limites $\mathrm{LI}(\mathrm{k})$ e $\mathrm{LS}(\mathrm{k})$ definem o intervalo de confiança bootstrap percentílico, com taxa de erro igual a $\alpha=0,05$, para $\mathrm{k}$ pontos amostrados por lavoura. A amplitude do intervalo de confianna bootstrap percentílico (AIC), para amostra de tamanho $\mathrm{k}$, é estimado por AIC $(\mathrm{k})=\mathrm{LS}(\mathrm{k})-\mathrm{LI}(\mathrm{k})$. O valor de $\mathrm{k}$, para o qual a AIC $(\mathrm{k})$ é igual ao AIC previamente admitido (por exemplo, AIC $=2$ percevejos), é o tamanho de amostra (número de pontos por lavoura), com 1- $\alpha=0,95$ de confiança. Para estes procedimentos estatísticos, foi usado o software R (R DEVELOPMENT CORE TEAM, 2014).

As densidades populacionais das espécies, para as coletas por pano-de-batida largo e rede entomológica, de diferentes cultivares e altitudes, foram comparadas pelo teste t bootstrap, usando o aplicativo Bio Estat 5.3 (AYRES et al., 2007). As demais análises estatísticas foram realizadas com o aplicativo Microsoft Office Excel.

\section{RESULTADOS E DISCUSSÃO}

Nas 100 lavouras estudadas, foram constatadas 16 cultivares de soja, das quais as oito mais frequentes estão listadas na tabela 1 . As cultivares 'BMX Potencia RR' (25\%) e 'Nidera 5009' (20\%) estiveram presentes em $45 \%$ das lavouras nas três safras do estudo. Em 39\% das lavouras, foram constatadas altitudes acima de $150 \mathrm{~m}$ (Tabela 2), correspondendo a três municípios (Itaara, Júlio de Castilhos e São Sepé). No geral, a altitude dos locais variou entre 55 e $494 \mathrm{~m}$, a latitude entre $29^{\circ} 15^{\prime}$ e $30^{\circ} 19^{\prime}$ e a longitude entre $53^{\circ} 50^{\prime}$ e $54^{\circ} 28^{\prime}$. Das lavouras avaliadas, $45 \%$ se encontravam no estágio reprodutivo. As densidades populacionais referentes às espécies Dichelops sp. e $\boldsymbol{P}$. guildinii e dois métodos de coleta foram maiores nas lavouras de menor altitude (altitude $<150 \mathrm{~m}$ ), sendo a densidade populacional de $\boldsymbol{E}$. heros não influenciada pela altitude nos dois métodos de coleta. Esse fato poderia ser explicado, em parte, pelos fatores abióticos, como a temperatura e umidade relativa do ar, que poderia afetar a abundância dos percevejos.

Excetuando Dichelops sp. na cultivar 'BMX Turbo RR' (Tabela 1), as densidades populacionais das três espécies de percevejos (Dichelops sp.; P. guildinii e $\boldsymbol{E}$. heros) não diferiu entre cultivares, para os dois métodos de coleta.

Algumas cultivares ('A8000' e 'Tijereta LT 2162') apresentaram densidades de percevejos menores do que o limite inferior do intervalo de confiança bootstrap percentílico, para as três espécies de percevejos e dois métodos de coleta. Essas inferioridades estão confundidas com os ambientes (altitude, manejo e outros) e a prática do controle de insetos diferenciados. Valores obtidos no pano-debatida largo discriminam mais as diferenças entre as cultivares, se comparados com a rede entomológica, possivelmente, por haver maior densidade populacional. A cultivar é um fator importante que deve ser considerado no manejo das pragas na cultura, pois influi diretamente na abundância e distribuição dos insetos. Nesse sentido, STÜRMER et al. (2014b) concluíram que os percevejos apresentaram maior densidade populacional na cultivar 'Fundacep 53 RR', quando comparada com a cultivar 'BMX Potência RR'.

Tabela 1 - Frequência de lavouras (Fo), média (número de percevejos $\mathrm{m}^{-2}$ ), com coleta por pano-de-batida largo e por rede entomológica, limites inferior (LI) e superior (LS) do intervalo de confiança bootstrap percentílico $(1-\alpha=0,95)$.

\begin{tabular}{|c|c|c|c|c|c|c|c|}
\hline \multirow{2}{*}{ Cultivar } & \multirow{2}{*}{$\begin{array}{l}\text { Fo } \\
(\%)\end{array}$} & \multicolumn{4}{|c|}{----Dichelops sp.--------- -------Piezodorus guildinii----' } & \multicolumn{2}{|c|}{---Euchistus heros---- } \\
\hline & & Pano & Rede & Pano & Rede & Pano & Rede \\
\hline 'BMX POTENCIA RR' & 25 & $0,32 \mathrm{a}^{*}$ & $0,03 \mathrm{~A}$ & $0,18 \mathrm{a}$ & $0,05 \mathrm{~A}$ & $0,17 \mathrm{a}$ & $0,02 \mathrm{~A}$ \\
\hline 'NIDERA 5009’ & 20 & $0,17 \mathrm{a}$ & $0,11 \mathrm{~A}$ & $0,29 \mathrm{a}$ & $0,24 \mathrm{~A}$ & $0,08 \mathrm{a}$ & $0,02 \mathrm{~A}$ \\
\hline 'CODETEC 231’ & 10 & $0,44 \mathrm{a}$ & $0,09 \mathrm{~A}$ & $0,49 \mathrm{a}$ & $0,38 \mathrm{~A}$ & $0,05 \mathrm{a}$ & $0,00 \mathrm{~A}$ \\
\hline 'A8000' & 10 & $0,09 \mathrm{a}$ & $0,03 \mathrm{~A}$ & $0,06 \mathrm{a}$ & $0,04 \mathrm{~A}$ & $0,09 \mathrm{a}$ & $0,02 \mathrm{~A}$ \\
\hline 'TIJERETA LT 2162' & 6 & $0,14 \mathrm{a}$ & $0,03 \mathrm{~A}$ & $0,06 \mathrm{a}$ & $0,07 \mathrm{~A}$ & $0,18 \mathrm{a}$ & $0,08 \mathrm{~A}$ \\
\hline 'CODETEC 5909' & 6 & $0,86 \mathrm{a}$ & $0,32 \mathrm{~A}$ & $0,40 \mathrm{a}$ & $0,06 \mathrm{~A}$ & $0,17 \mathrm{a}$ & $0,04 \mathrm{~A}$ \\
\hline 'BMX TURBO RR' & 6 & $0,70 \mathrm{a}$ & $0,07 \mathrm{~A}$ & $0,24 \mathrm{~b}$ & $0,07 \mathrm{~A}$ & $0,26 \mathrm{~b}$ & $0,02 \mathrm{~A}$ \\
\hline 'NIDERA 6411' & 5 & $0,54 \mathrm{a}$ & $0,06 \mathrm{~A}$ & $0,28 \mathrm{a}$ & $0,11 \mathrm{~A}$ & $0,29 \mathrm{a}$ & $0,02 \mathrm{~A}$ \\
\hline Média & - & 0,35 & 0,08 & 0,27 & 0,11 & 0,28 & 0,05 \\
\hline LI & - & 0,24 & 0,05 & 0,16 & 0,06 & 0,11 & 0,01 \\
\hline LS & - & 0,45 & 0,11 & 0,38 & 0,17 & 0,49 & 0,10 \\
\hline
\end{tabular}

*Médias seguidas de letra diferente na linha, minúscula para pano e maiúscula para rede, diferem significativamente pelo teste t $(\alpha<0,05$; 4.000 reamostras). 
Tabela 2 - Número médio de percevejos $\mathrm{m}^{-2}$, de acordo com a espécie, com o método de coleta e com a altitude da lavoura.

\begin{tabular}{|c|c|c|c|c|}
\hline \multirow{2}{*}{ Espécie de percevejo } & \multirow[b]{2}{*}{ Pano } & \multirow[b]{2}{*}{ Rede } & \multirow{2}{*}{ Pano } & \multirow{2}{*}{ Rede } \\
\hline & & & & \\
\hline Dichelops sp. & $0,47 \mathrm{a}^{*} \mathrm{~A}$ & $0,11 \mathrm{aA}$ & $0,17 \mathrm{aB}$ & $0,02 \mathrm{aB}$ \\
\hline Piezodorus guildinii & $0,39 \mathrm{aA}$ & $0,17 \mathrm{aA}$ & $0,09 \mathrm{aB}$ & $0,03 \mathrm{aB}$ \\
\hline Euchistus heros & $0,36 \mathrm{aA}$ & $0,08 \mathrm{aA}$ & $0,12 \mathrm{aA}$ & $0,02 \mathrm{aA}$ \\
\hline
\end{tabular}

*Médias não seguidas por mesma letra (minúscula na vertical e maiúscula entre altitude dentro de método) diferem pelo teste $\mathrm{t}(\alpha<0,05 ; 4.000$ reamostras).

Pelas estimativas por intervalo (LI e LS), na média das cultivares, a densidade populacional resultante da coleta por pano-de-batida largo foi significativamente superior a da rede entomológica para duas (Dichelops sp. e E. heros) das três espécies (Tabela 1). Deve ser lembrado que a comparação da área ou volume de plantas cobertas pelos dois métodos de coleta é dificultada, pois o resultado da coleta da rede depende da fuga dos percevejos adultos, da repetibilidade dos movimentos do operador e, possivelmente, do horário de coleta, devido à posição dos insetos dentro do dossel das plantas.
As médias da densidade populacional, por classe de densidade, e o tamanho de amostra para uma amplitude do intervalo de confiança bootstrap percentílico (AIC) igual a um $(\mathrm{AIC}=1)$ e dois $(\mathrm{AIC}=2)$ percevejos por ponto, de cada espécie e método de coleta, são apresentadas na tabela 3. Considerando as três espécies de percevejo, observa-se frequência média de $37 \%$ das lavouras sem percevejos, pelo método do pano-de-batida largo, e 59\% com o método da rede entomológica. Este fato repercute na baixa estimativa da média da densidade populacional dos percevejos. A baixa densidade populacional, também

Tabela 3 - Frequências de lavouras (Fo), média da densidade populacional (percevejos $\mathrm{m}^{-2}$ ) e tamanho de amostra para a amplitude do intervalo de confiança bootstrap percentílico (AIC), de acordo com a espécie, com o método de coleta e com diferentes classes da densidade populacional (Classe, percevejos $\mathrm{m}^{-2}$ ).

\begin{tabular}{|c|c|c|c|c|c|c|c|c|}
\hline \multirow{2}{*}{ Classe } & \multicolumn{4}{|c|}{--Pano-de-batida largo------------------' } & \multicolumn{4}{|c|}{---Rede entomológica------- } \\
\hline & Fo & Média & $\mathrm{AIC}=1 \dagger^{1}$ & $\mathrm{AIC}=2$ & Fo & Média & $\mathrm{AIC}=1$ & $\mathrm{AIC}=2$ \\
\hline \multicolumn{9}{|c|}{ 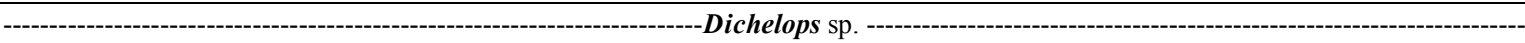 } \\
\hline 0 & 26 & 0 & - & - & 52 & 0 & - & - \\
\hline 0,01 a 0,50 & 54 & 0,16 & 6,4 & 4,1 & 45 & 0,12 & 5,5 & 4,0 \\
\hline 0,51 a 1,00 & 8 & 0,74 & 38,6 & 9,2 & 2 & 0,60 & 12,5 & 4,0 \\
\hline 1,01 a 1,50 & 7 & 1,28 & 43,4 & 12,1 & 1 & 1,43 & 40,0 & 11,0 \\
\hline 1,51 a 2,00 & 3 & 1,68 & 49,7 & 13,0 & - & - & - & - \\
\hline$>2,00$ & 2 & 3,15 & 91,50 & 24,5 & - & - & - & - \\
\hline 0 & 44 & 0 & P & $\begin{array}{c}\text { orus gui } \\
-\end{array}$ & 54 & 0 & - & - \\
\hline 0,01 a 0,50 & 43 & 0,21 & 11,0 & 4,6 & 42 & 0,12 & 6,4 & 4,0 \\
\hline 0,51 a 1,00 & 6 & 0,66 & 26,0 & 6,8 & 0 & - & - & - \\
\hline 1,01 a 1,50 & 1 & 1,13 & 83,0 & 21,0 & 3 & 1,20 & 101,7 & 26,7 \\
\hline 1,51 a 2,00 & 4 & 3,12 & 167,5 & 77,0 & 0 & - & - & - \\
\hline$>2,00$ & 0 & - & - & - & 1 & 2,6 & $>200$ & 53,0 \\
\hline 0 & 42 & 0 & - & - & 72 & 0 & - & - \\
\hline 0,01 a 0,50 & 48 & 0,13 & 5,5 & 4,0 & 26 & 0,09 & 5,2 & 4,0 \\
\hline 0,51 a 1,00 & 6 & 0,77 & 36,2 & 8,8 & 1 & 0,57 & 18,0 & 4,0 \\
\hline 1,01 a 1,50 & 2 & 1,38 & 57,0 & 15,0 & - & - & - & - \\
\hline$>2,00$ & 2 & 6,95 & 161,0 & 115,5 & 1 & 2,57 & 76,0 & 21,0 \\
\hline
\end{tabular}

$\dagger^{1 /}$ Amplitude do intervalo de confiança bootstrap percentílico (AIC, $\left.1-\alpha=0,95\right)$ igual a um $(\mathrm{AIC}=1)$ ou dois $(\mathrm{AIC}=2)$ percevejos.

Ciência Rural, v.46, n.3, mar, 2016. 
relatada por STÜRMER et al. (2014a), em alguns estágios de desenvolvimento da cultura, pode ser devido à frequência de controle das pragas praticada pelos produtores.

Para as três espécies e os dois métodos de coleta, com o acréscimo da média (classes de densidade populacional), há aumento do tamanho de amostra. Essa relação se deve à forma de quantificar o erro de estimação (AIC) em número de percevejos. No caso em que essa amplitude é atribuída como sendo uma proporção da média da densidade populacional (STÜRMER et al., 2014a), esta relação é inversa, ou seja, menores densidades populacionais implicam maiores tamanhos de amostra.

Considerando-se uma lavoura com classe de densidade populacional igual a 1,51 a 2,00 percevejos $\mathrm{m}^{-2}$ como sendo um valor próximo ao nível de dano econômico e sendo o erro de estimação igual a dois percevejos $(\mathrm{AIC}=2)$, o tamanho de amostra é igual a 13 para Dichelops sp., 77 para P. guildinii e 15 para $\boldsymbol{E}$. heros, usando o pano-de-batida largo como método de coleta (Tabela 3). Para estes valores de tamanho de amostra, o erro de estimação usado $(\mathrm{AIC}=2)$ em percentagem da média é, respectivamente, 119, 64 e 145\%. Estes percentuais de erro são superiores aos relatados (10 a 50\%) no estudo de STÜRMER et al. (2014a).

Para adultos da espécie Dichelops sp., usando erro de estimação de $119 \%$, STÜRMER et al. (2014a) teriam estimado tamanho de amostra igual a $64,15,7$ e 7 pontos, respectivamente, nos estágios R6, R7.1, R7.3 e R8.2. Esses valores são próximos ao estimado neste estudo, 13 pontos. Para adultos da espécie $\boldsymbol{P}$. guildinii, usando erro de estimação igual a $64 \%$, STÜRMER et al. (2014a) teriam estimado tamanho de amostra igual a 191, 94, 42 e 6 pontos, respectivamente, nos estágios R1, R4, R6 e R8.2. Esses valores são mais variados, mas estão próximos ao tamanho de amostra estimado neste estudo para a mesma espécie, 77 pontos.

Como o tamanho de amostra varia com a média da densidade populacional e esta com os estágios de desenvolvimento da cultura e outros fatores ambientais, não é possível decidir por um tamanho de amostra adequado para qualquer condição. Em classe de densidade populacional menor, há redução do tamanho de amostra. Dessa forma, para pequenas densidades populacionais, o tamanho de amostra é pequeno ou até desnecessário, pois poucos pontos amostrados seriam suficientes para decidir sobre o controle da praga.

Variações do tamanho de amostra para estimar a densidade populacional de percevejos foram relatados para a cultura dasoja (STÜRMER at al., 2014a, 2014b), algodão (REAY-JONES et al., 2009), arroz (ESPINO et al., 2008) e tomate (CULLEN et al., 2000). Uma possível razão das diferenças no tamanho de amostra é a distinta mobilidade das espécies e a consideração ou não das ninfas na contagem. COSTA \& LINK (1982) mencionaram que $\boldsymbol{P}$. guildinii apresenta maior dispersão que a espécie $\boldsymbol{E}$. heros.

\section{CONCLUSÃO}

O tamanho de amostra (número de pontos) para determinar a densidade populacional de percevejos varia com o método de coleta e a densidade populacional.

Usando o pano-de-batida largo para a coleta de percevejos em soja, para um erro de estimação (amplitude do intervalo de confiança bootstrap percentílico) igual a dois percevejos e densidade populacional na classe de 1,5 a 2,0 percevejos $\mathrm{m}^{-2}$, o tamanho de amostra é $13,77 \mathrm{e}$ 15, respectivamente, para as espécies Dichelops sp., P. guildinii e E. heros.

Usando a rede entomológica para a coleta de percevejos em soja, para erro de estimação igual a dois percevejos e densidade populacional na classe de 1,0 a 1,5 percevejos $\mathrm{m}^{-2}$, o tamanho de amostra é 11 , 27 e 4, respectivamente, para as espécies Dichelops sp., P. guildinii e E. heros.

\section{AGRADECIMENTOS}

Ao Conselho Nacional de Desenvolvimento Científico e Tecnológico (CNPq), pelas bolsas de produtividade, e à Coordenação de Aperfeiçoamento de Pessoal de Nível Superior (CAPES), pela bolsa do programa PEC-PG.

\section{REFERÊNCIAS}

AYRES, M. et al. BioEstat - Aplicações estatísticas nas áreas das ciências biomédicas. 2007. Disponível em: $<$ http://rs579.rapidshare. com/files/171642982/BioEstat.zip>. Acesso em: 23 fev. 2014.

BARBOSA, J.C. Métodos estatísticos aplicados à entomologia. Jaboticabal: DCE/ FCAV/UNESP, 2001. 250p.

CONAB (COMPANHIA NACIONAL DE ABASTECIMENTO). $8^{\circ}$ Levantamento Maio 2015. In: Monitoramento agrícola: cultivo de verão. Disponível em: <http://www.conab.gov.br>. Acesso em: 04 jun. 2015.

CORREAA-FERREIRA, B. Amostragem de pragas da soja.In: HOFFMANN-CAMPO, C. et al. (Eds.). Soja: manejo integrado de insetos e outros artrópodes-praga. Brasília: EMBRAPA, 2012. p.631-672. Disponível em: <http://www.cnpso.embrapa.br/ artropodes/Capitulo9.pdf>. Acesso em: 04 jun. 2015.

CORRÊA-FERREIRA, B.S.Suscetibilidade da soja a percevejos na fase anterior ao desenvolvimento das vagens. Pesquisa

Ciência Rural, v.46, n.3, mar, 2016. 
Agropecuária Brasileira, v.40, p.1067-1072, 2005. Disponível em: <http://dx.doi.org/10.1590/S0100-204X2005001100003>. Acesso em: 04 jun. 2015.

COSTA, E.C.; LINK, D. Dispersão de adultos de Piezodorus guildinii e Nezaraviridula (Hemiptera: Pentatomidae) em soja. Revista do Centro de Ciências Rurais, v.12, p.51-57, 1982. Disponível em: $<$ http://coral.ufsm.br/revistaccr/index.php/RCCCR/ article/view/441/439>. Acesso em: 04 jun. 2015.

CULLEN, E. et al. Quantifying trade-offs between pest sampling time and precision in commercial IPM sampling programs. Agricultural Systems, v.66, p.99-113, 2000. Disponível em: $<$ http:// www.sciencedirect.com/science/article/pii/S0308521X0000038X>. Acesso em: 04 jun. 2015.

EFRON, B. Bootstrap method: another look at the jackknife. Annals of Statistics, v.7, p.1-26, 1979.

ESPINO, L. et al. Determination of Oebaluspugnax (Hemiptera: Pentatomidae) spatial pattern in rice and development of visual sampling methods and population sampling plans. Journal of Economic Entomology. v.101, p.216-225, 2008. Disponível em: $<$ http://dx.doi.org/10.1603/0022-0493(2008)101[216:DOOPHP]2. 0.CO;2>. Acesso em: 04 jun. 2015.

FARIAS, P. et al. Distribuição espacial da lagarta-do-cartucho, Spodoptera frugiperda (J.E. Smith) (Lepidoptera: Noctuidae), na cultura do milho. Neotropical Entomology, v.30, p.681689, 2001. Disponível em: <http://dx.doi.org/10.1590/S1519566X2001000400025>. Acesso em: 04 jun. 2015.

FERREIRA, D.F. Estatística básica. Lavras: UFLA, 2005. 664p.

GALLO, D. et al. Entomologia agrícola. Piracicaba: Fealq, 2002. 920p.

GUEDES, J. et al. Capacidade de coleta de dois métodos de amostragem de insetos-pragas da soja em diferentes espaçamentos entre linhas. Ciência Rural, v.36, p.1299-1302, 2006. Disponível em: <http://dx.doi.org/10.1590/S0103-84782006000400040>. Acesso em: 04 jun. 2015.
LARA, R. et al. Amostragem, diversidade e sazonalidade de Hemerobiidae (Neuroptera) em Coffea arábica L. cv. 'Obatã' (Rubiaceae). Revista Brasileira de Entomología, v.52, p.117123, 2008. Disponível em: <http://dx.doi.org/10.1590/S008556262008000100020>. Acesso em: 04 jun. 2015.

PANIZZI, A.; CORRÊA-FERREIRA, B. Comparação de dois métodos de amostragem de artrópodos em soja. Anais da Sociedade Entomológica do Brasil, v.7, p.60-66, 1978.

R DEVELOPMENT CORE TEAM. R: a language and environment for statistical computing. Vienna: R Foundation for Statistical Computing, 2014. Disponível em: <http://www.Rproject.org $>$. Acesso em: 04 jun. 2015.

REAY-JONES, F. et al. Sampling stink Bugs (Hemiptera: Pentatomidae) for population estimation and Pest Management in Southeastern cotton production. Journal of Economic Entomology, v.102, n.6, p.2360-2370, 2009. Disponível em: <http://dx.doi. org/10.1603/029.102.0643>. Acesso em: 04 jun. 2015.

STORCK, L. et al. A sampling procedure for quantifying mites in soybeans. Experimental \& Applied Acarology, v.57, n.2, p.117-126, 2012. Disponível em: <http://link.springer.com/ article/10.1007/s10493-012-9547-8>. Acesso em: 04 jun. 2015.

STÜRMER, G.R. et al. Sample size for estimating the population of stink bugs in soybean crops. Revista Ciência Agronômica, v.45, n.1, p.155-167, 2014a. Disponível em: <http://dx.doi.org/10.1590/ S1806-66902014000100019>. Acesso em: 04 jun. 2015.

STÜRMER, G.R. et al. Eficiência do pano-de-batida na amostragem de insetos-praga de soja em diferentes espaçamentos entre linhas e cultivares. Semina: Ciências Agrárias, v.35, n.3, p.1177-1186, 2014 b. Disponível em: <http://www.uel.br/revistas/uel/index.php/semagrarias/ article/viewFile/13720/pdf_316>. Acesso em: 04 jun. 2015.

STÜRMER, G.R.et al. Eficiência de métodos de amostragem de lagartas e de percevejos na cultura de soja. Ciência Rural, v.42, p.2105-2111, 2012. Disponível em: <http://dx.doi.org/10.1590/ S0103-84782012005000145>. Acesso em: 04 jun. 2015. 